\title{
Detection of Face Orientation by 3D Measurement of Facial Features
}

\author{
Member \\ Ming XU \\ Non-member \\ Takao AKATSUKA \\ (Yamagata University) \\ (Yamagata University)
}

\begin{abstract}
An approach to detect human facial orientation by reconstructing the three-dimensional position of facial points from stereo images is proposed. The purpose is to develop a flexible and accurate facial orientation detection method for human interaction with computers. Four facial points (pupils and mouth corners) are extracted using a global-to-local method and their three-dimensional coordinates are reconstructed from stereo images. Facial orientation is obtained by calculating the normal direction of the plane formed by three facial feature points. The advantages of the proposed method are first, that it is flexible and easy to adapt to different persons and circumstances; and second, that it eliminates the need for prior knowledge of image vs. model correspondence or the physical parameters of facial features. Analytical and experimental results show that the proposed method performs well when the head is not far away from the frontal view. These characteristics are especially useful for constructing a human-computer interaction system.
\end{abstract}

Key words: facial orientation estimation, facial feature detection, stereo vision, HCI

\section{Introduction}

With the rapid growth of computer power in recent years, the need for a natural computer-human interaction system has grown larger. The orientation of a person's face is potentially very useful for determining components of human-computer interaction. Many applications using face orientation have been reported so far. For example, facial orientation is used as pointing tool to activate a window at which the user is looking[1]. Corresponding to the viewer's facial orientation to the screen, the computer displays a different projection of a scene, giving the illusion of 3-D video[2]. To implement a very low bandwidth visual communication, facial orientation is transmitted and used to synthesize the face which is viewed remotely[3]. By tracking the change of facial orientation, a head sign based interface is helpful for physically handicapped people. In addition, the application for face recognition is also proposed[4].

Many methods have been proposed for head pose detection, and vision based methods have attracted the most interests from researchers for their non-intrusiveness. Vision based methods usually capture the head image by camera, and estimate head pose through image processing. We classify these methods into four groups. The first consists of the image based methods which use the correlation between input image and images in database to determine the pose of head in input image. A database is constructed either by taking pictures of the head corresponding to all possible poses and lighting conditions or by projecting the 3-D head model to obtain faces with different poses[5,6]. These methods require a large amount of disk space to save the database and lack flexibility in adapting to different persons or illumination conditions. The second group consists of the geometry based methods which use the geometrical property of face, such as the gravitational center of the face[7], the distance between facial features[8], or eye width and nose length[9]. These methods all have a common problem in that their accuracy clearly tends to decrease when the face is approaching the frontal view. The third of these groups consists of the model based methods which exploit prior knowledge of head models and require that at least three corresponding points between image and 3D head model be previously known $[10,11]$. These methods usually need manual operation to provide the 3-D coordinates of facial features on the model. 
The fourth and last of these groups are those methods which require the application of auxiliary marks with certain shapes and colors to a person's face and then estimate facial orientation from the variation in the shape of the marks[12].

In this paper, we describe a non-intrusive facial orientation detection method derived from the measurement of the 3-D coordinates of facial features from input stereo images. The purpose is to develop a flexible and accurate facial orientation detection method that will prove useful for the construction of a pose-based human-computer interaction system. First, we describe a global-to-local method for detecting four facial points (pupils and mouth corners) from the input images. Then the 3-D positions of facial feature points are obtained directly using the stereo vision method. And finally, facial orientation is obtained by calculating the normal direction of the plane formed by three facial feature points. The advantages of this method are first, it is flexible and does not require a pre-produced face database and therefore is easily adapted to different persons and circumstance; and second, it eliminates the need for prior knowledge of image vs. model correspondence or the physical parameters of facial feature. Moreover, the processing is simple and thus it is possible to execute at a high rate of speed. Analytical and experimental results show that accuracy is higher when the face is not far away from the frontal area. This would be a good property for human-computer interaction applications.

\section{Detection of Facial Features}

\subsection{Selection of Features}

It is intuitive that the orientation of a plane can be determined from the 3-D position of three non-collinear points in that plane, and it is generally true that the pupils and mouth corners are coplanar, and that the plane they form best reflect frontal face orientation (except the cases of extreme changes of expression). Although other facial points, such as nostrils and the shadow of lower lip can also be used, their positions are prone to be affected by change in lighting conditions and poses. As long as the 3-D position of the pupils and mouth corners are known, facial orientation can be determined by computing the normal direction of the triangle formed by the pupils and mouth corners.(See Fig.1).

We take a global-to-local approach for detecting the facial features. First, we search the area of the face and then search out the facial features from a restricted region inside the face. The only circumstantial assumptions we make are that the background geometry and texture are static or are only subject to low frequency changes, and that lighting

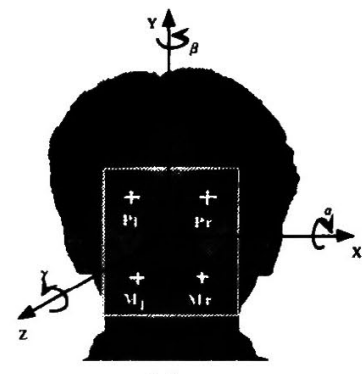

(a)

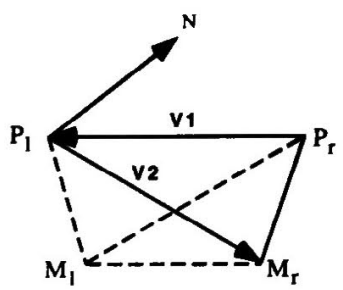

(b)
Fig. 1 Facial Features and Face Orientation

conditions are controllable. It is relatively easy to satisfy these constraints in any indoor situation. It is also feasible to assume that head rotation is restricted within $\mathbf{3 0}$ degrees from the frontal view position, because facial orientations in this range are most useful for applications of humancomputer interactions.

\subsection{Detection of Facial Area}

The person is segmented from the input image through the use of a background subtraction scheme. It is therefore reasonable to take the input as a head-with-shoulder image, since the head area can thus be extracted approximately by comparing the horizontal size difference between head and shoulders. The facial area usually has a higher gray level, while the hair area has a very low gray level. The facial area is then extracted by thresholding the head area with an automatic threshold selection method[13]. To remove the holes cause by dark points in the face (i.e., the pupils), a morphological opening operation is applied, and the facial area is cleanly extracted. This process is shown in Fig.2.

\subsection{Detection of the Pupils}

The eye region is predicted to be in the top half of the exterior rectangle of the face, and the pupils are found in the left and right part respectively. The pupils are usually the darkest regions in the facial area, so we can search for the pupils by thresholding and searching for two darkest points in the eye region. During the thresholding operation, the

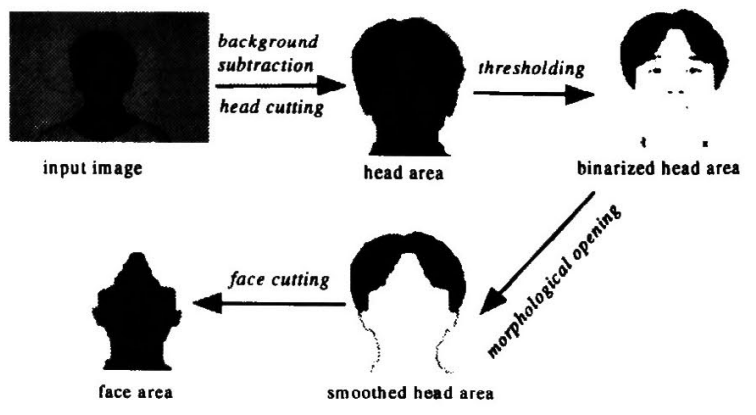

Fig. 2 Facial Area Detection 


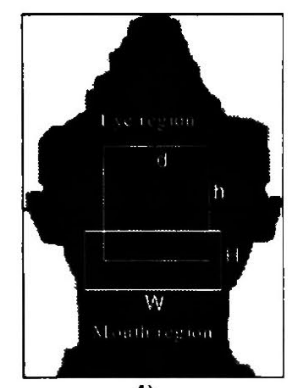

(b)

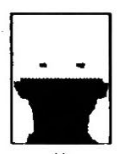

(a)

eye region thresholding

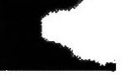

(d)

horizontal projectio of moush region

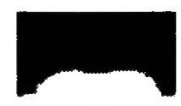

(c)

vertical projection of mouth region

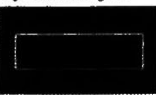
mourh region
Fig. 3 Facial Feature Detection

pupil may be divided into several points and this may cause uncertainty as to the pupil's position. To prevent this problem, we first do a morphological dilation operation using a $3 \times 3$ square structural element in order to enlarge the dark pupil region. Because the appropriate threshold value may change for different people or under different lighting conditions, a fixed threshold value is not suitable. In this case, we adopt an iterative thresholding method which thresholds the eye region until the number of dark pixels in both part of the eye region are less than a predefined value. The dark pixels form two dark blobs in the eye region, and the pupil positions are determined as the center of the detected blobs. Since the thresholding value is adjusted automatically, it is possible with this method to find pupils on faces illuminated by different intensities of light. This is shown in Fig.3(a).

\subsection{Detection of Mouth Corners}

From the geometrical property of the human face, the approximate region of the mouth can be predicted from the position of the pupils and the distance between the pupils. As shown in Fig.3(b), if the coordinates of two pupils are $\left(x_{1}, y_{1}\right)$ and $\left(x_{r}, y_{r}\right)$, then we have

$$
\begin{aligned}
& d=\left|x_{r}-x_{1}\right| \\
& Y=\mid y_{1}+y_{r} / 2
\end{aligned}
$$

where $d$ is the horizontal distance between two pupils, and $\mathrm{Y}$ is the average vertical coordinate of two pupils.

We suppose here the mouth region is represented by a rectangle whose size is represented as $\mathrm{W} \times \mathrm{H}$ and the coordinates of its center as $\left(\left(x_{1}+x_{r}\right) / 2,(Y+h)\right)$. So the coordinates of the rectangle, i.e. $\left(X_{l}, Y_{u}\right),\left(X_{l}, Y_{d}\right),\left(X_{r}, Y_{u}\right)$ and $\left(X_{r}, Y_{d}\right)$, can be decided as follows,

$$
\begin{aligned}
& X_{1}=\left(x_{1}+x_{\mathrm{r}}\right) / 2-W / 2 \\
& X_{1}=\left(x_{1}+x_{\mathrm{r}}\right) / 2+W / 2 \\
& Y_{u}=(Y+h)-H / 2 \\
& Y_{d}=(Y+h)+H / 2
\end{aligned}
$$

To determine the relation between $h, W, H$ and $d$, we use the statistical characteristics obtained from the sample image set, which consisted of 35 facial images. We then have:

$$
\mathrm{h}=1.1 \mathrm{~d}, \mathrm{~W}=0.95 \mathrm{~d}, \mathrm{H}=0.42 \mathrm{~d}
$$

In order to deal variations in facial shape, $\mathrm{W}$ and $\mathrm{H}$ are set to larger value as follows,

$$
\mathrm{W}=1.4 \mathrm{~d}, \mathrm{H}=0.7 \mathrm{~d}
$$

By calculating the gray value integration projection along horizontal and vertical direction respectively, a small rectangle that covers the mouth is extracted by thresholding the respective projection value. When teeth are visible, the distribution of integration value may be violated, so we perform a similar morphological dilation operation as above to expand the dark pixels of the mouth before doing the projection. The horizontal position of the mouth corners are obtained from the horizontal coordinates of the extracted rectangle but are adjusted by adding or subtracting $\mathbf{N}$ pixels for the left and right corners respectively, where $\mathrm{N}$ shows how many times the dilation operation is done. In our experiment, we iterate the dilation three times. The vertical position of the mouth corners is determined as the position with the lowest gray value in the column of the detected rectangle, as shown in Fig.3(c), (d), (e).

\subsection{Accuracy of Feature Detection}

We have tested the feature detector on pictures of thirtyfive different people taken indoors. All the test faces are approximately frontally and vertically orientated. Of these thirty-five pictures, thirty-one are of men, and four are of women. In five of the pictures the people are wearing glasses. Comparing the detector output with the manual detection result, the larger coordinate difference of the absolute value of $x$ and $y$ is defined as the detector error. Among the test pictures, the range of error is from one to three pixels with an average error of 1.8 pixels.

\section{Stereo Vision Based Pose Detection}

\subsection{3-D Measurement of Feature Points}

In order to find the 3-D position of the detected feature points, we adopt the stereo vision method[15]. We set stereo

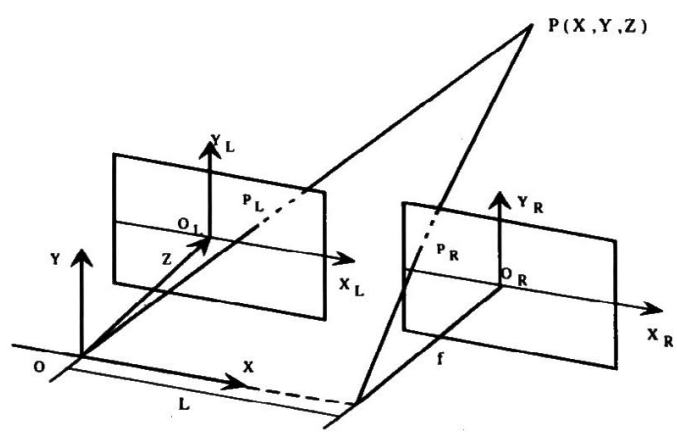

Fig. 4 Stereo Camera Configuration 
cameras in the parallel manner, as shown in Fig.4. The parallel configuration is simple, and it makes searching the four corresponding points in the stereo pair image a fairly easy task. The global coordinate system $\mathrm{O}-\mathrm{X}-\mathrm{Y}-\mathrm{Z}$ is set in accordance with the coordinate system of the left camera but shifted by focal length $f$ in $\mathrm{Z}$ axis. The distance between the optical center of the two cameras is $L$. The relation between the three-dimensional feature point $\mathrm{P}(\mathrm{X}, \mathrm{Y}, \mathrm{Z})$ and its projection on the left and right image planes $P_{1}\left(x_{1}, y_{1}\right)$ and $P_{r}\left(x_{r}, y_{r}\right)$ is shown in Fig.4. From triangular geometry and camera parameters, we get:

$$
(X, Y, Z)^{T}=\frac{L \mu}{D_{p}}\left(x_{1}, y_{1}, f\right)^{T}
$$

where $D_{p}$ is the disparity of $\mathrm{P}$ between left and right images and is calculated as follows:

$$
D_{p}=x_{l}-x_{r}
$$

$\mu$ is the resolution ( pixel/mm) of camera's CCD sensor.

\subsection{Face Orientation Calculation}

The orientation of the triangle formed by the pupils and mouth corners can be expressed by its normal direction. Although any one of the four triangles (e.g., in Fig. 1(b), $\mathrm{P}_{1}$ $P_{r}-M_{1}, P_{1}-P_{r}-M_{r}, P_{1}-M_{r}-M_{1}$ and $P_{r}-M_{r}-M_{l}$ ) can be used, they should be combined in the same direction (e.g., clockwise) so as to maintain a consistent normal direction.

In Fig.1(b), suppose the 3-D coordinates of point $P_{l}, P_{r}$, and $M_{r}$ are $(a 1, b 1, c 1),(a 2, b 2, c 2)$ and $(a 3, b 3, c 3)$ respectively, then the normal vector of triangle $P_{1}-P_{r}-M_{r}$ is:

where

$$
\mathbf{N}=a \hat{x}+b \hat{\mathbf{y}}+c \hat{z} \quad \ldots \ldots \ldots \ldots \ldots \ldots(7)
$$

$$
\begin{aligned}
& a=b_{1} c_{3}+b_{2} c_{1}+b_{3} c_{2}-b_{1} c_{2}-b_{2} c_{3}-b_{3} c_{1} \\
& b=a_{1} c_{2}+a_{2} c_{3}+a_{3} c_{1}-a_{1} c_{3}-a_{2} c_{1}-a_{3} c_{2} \\
& c=a_{1} b_{3}+a_{2} b_{1}+a_{3} b_{2}-a_{1} b_{2}-a_{2} b_{3}-a_{3} b_{1}
\end{aligned}
$$

$\hat{\mathbf{x}}, \hat{\mathbf{y}}, \hat{\mathbf{z}}$ are unit vectors, which direct to the $\mathrm{X}, \mathrm{Y}, \mathrm{Z}$ axes of the global coordinates system respectively.

Usually, it is intuitive to express head pose by the rotation angle around $\mathrm{X}, \mathrm{Y}$, and $\mathrm{Z}$ axis (expressed as $\alpha, \beta$ and $\gamma$ respectively), by which the head rotates from the original direction (direction of $\mathrm{Z}$ ) to the present direction. $\alpha$ and $\beta$ are equivalent to the angle formed by normal vector with $\mathrm{X}-\mathrm{O}-\mathrm{Z}$ plane and $\mathrm{Y}-\mathrm{O}-\mathrm{Z}$ plane respectively:

$$
\begin{aligned}
& \alpha=\arcsin \frac{b}{\sqrt{b^{2}+c^{2}}} \\
& \beta=\arcsin \frac{a}{\sqrt{a^{2}+c^{2}}}
\end{aligned}
$$

Because $r$ expresses the rotation parallel to the image plane, it can be directly calculated from the 2-D coordinate of the pupils:

$$
\gamma=\arctan \frac{Y_{p 2}-Y_{p 1}}{X_{p 2}-X_{p 1}}=\arctan \frac{y_{i p 2}-y_{i p 1}}{x_{i p 2}-x_{i p 1}} \cdots \ldots \ldots
$$

where $x_{1 p 1}, y_{1 p 1}$ and $x_{1 p 2}, y_{1 p 2}$ are the 2-D coordinates of the pupils in the left image.

\section{Performance Analysis}

We observed that the accuracy of head pose detection has close relation to head pose in general. In order to find the detection accuracy and its relation to head pose, we adopt a sphere model with radius $\mathrm{R}$ for approximation of the head. In the sequent part of the section, we will analyze from two aspects the relation between the rotation angle detection accuracy of a point and its position in the sphere.

\subsection{The Least Sensitive Angle}

As shown in Fig.5, we consider the case of a head rotating around the $\mathrm{Y}$-axis (the situation of a head rotating around the $\mathrm{X}$-axis is similar). When a head rotates, the projective position of feature point $\mathrm{F}$ in the image plane also changes. When $F$ moves to a position where its projective point in the image plane reaches the furthest position in $\mathrm{X}$ direction, the feature rotation around this position produces almost no change to the $\mathrm{X}$ coordinate, thus causing an error in the position of the detected feature. We express this position as an angle and call it the least sensitive angle. Its value is affected by the radius of the head model, the distance from the head to the camera and the distance from the head rotation axis to the camera optical axis. The case of the head rotation around the Y-axis as shown in Fig.5, can be calculated as:

$$
\theta=\varphi_{1}+\varphi_{2}
$$

where

$$
\varphi_{1}=\arctan \frac{L / 2}{Z+f}
$$

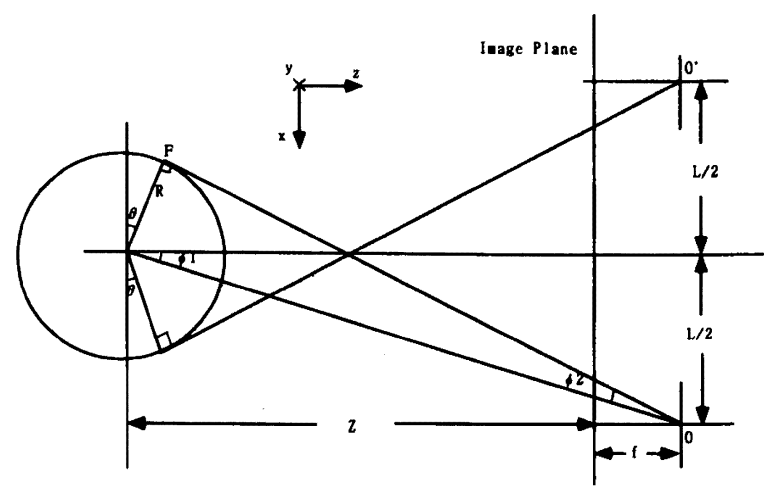

Fig. 5 Illustration of the Least Angle 


$$
\varphi_{2}=\arcsin \frac{R}{\sqrt{(Z+f)^{2}+(L / 2)^{2}}}
$$

\subsection{Camera Depth Resolution}

Head rotation which brings a change in depth of the target point will directly affect the Z-coordinate of feature points. Therefore, the depth resolution of the stereo camera also influences the accuracy of head pose estimation. We define the depth resolution as the depth variation caused by disparity variation of one pixel as in (17). $\Delta \mathrm{Z}$ is the depth resolution of stereo camera (in $\mathrm{mm} / \mathrm{pixel}$ ), $d$ is the disparity of feature point between left and right image, $f$ is the focal length of camera, $L$ is the length between two camera centers, and $\mu$ is the resolution of camera's CCD sensor.

$$
\Delta Z=\frac{L f \mu}{d}-\frac{L f \mu}{d+1}=\frac{L f \mu}{d(d+1)} \approx \frac{L f \mu}{d^{2}} \ldots \ldots
$$

If we replace $d$ by (5), we have

$$
\Delta Z=\frac{Z^{2}}{L f \mu}
$$

Let us assume one point in the sphere rotating around the Yaxis, its position represented by $\theta$, which is the rotation angle from the $\mathrm{Z}$-axis. When the point rotated an angle of $\Delta \theta$, the change of depth is estimated as follows:

$$
\Delta Z=R(\cos \theta-\cos (\theta+\Delta \theta))
$$

We suppose the radius $R$ to be $65 \mathrm{~mm}$ in which formula (19) is depicted as Fig.6(a). The three corresponding curves takes the values of 1,3 , and 5 degrees respectively. This graph shows the depth change when a feature point rotates a given angle at different positions. It also implies the necessary depth resolution of the stereo camera for detecting the given rotation angle.

Using the depth resolution obtained from (17), we changed the unit of the vertical axis in Fig.6(a) from millimeters to pixels, and we arrived at Fig.6(b). This graph shows the disparity corresponding to a given rotation angle at different positions. It also implies the 2D point's location accuracy required for detecting a certain rotation of the point. The two graphs exhibit an identical fact from two different aspects, namely that when feature point is in the direction of $\mathbf{Z}$, the system resolution required for detecting certain rotation is the highest. Or to put it another way, the rotation detection accuracy around the $\mathrm{Z}$ direction is the lowest.

\subsection{Position with High Detection Accuracy}

Based on the analysis in section 4.1 and 4.2, we know that when the face is frontally oriented, none of the four feature points (pupils and mouth corners) are found in either

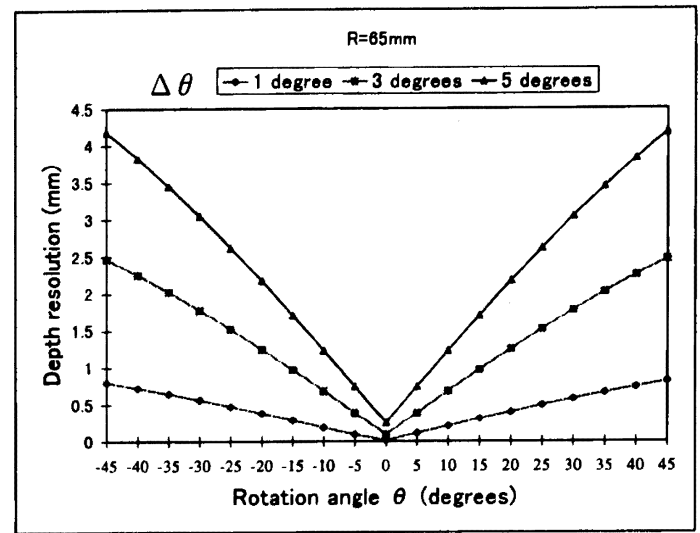

Fig. 6(a) Camera Depth Resolution

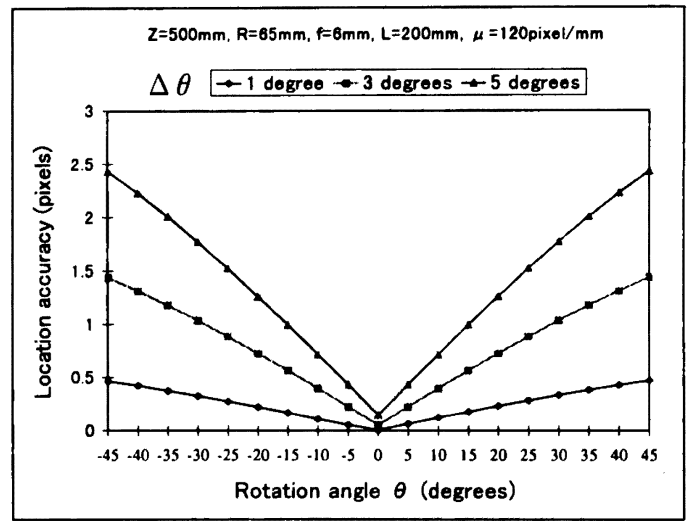

Fig. 6(b) Feature Location Accuracy

of the two regions, neither the region around the least sensitive angle nor the region around the Z-axis, where the detection accuracy of the rotation angle degrades most. So we can conclude that when face is not far away from the frontal view, the pose detection accuracy is high.

The above analysis not only reveals the relation between accuracy and position, but also predicts possible directions for improving system performance. For example, as shown in (15), (16) and (18), a higher system performance can be obtained by properly adjusting the inner parameters of the camera.

\section{Experimental Results}

\subsection{Virtual Camera Experiments}

In order to evaluate the performance of the proposed method, we first experimented with virtual stereo cameras. A pre-produced CG head model is utilized, and the frontal facial image of the subject is mapped to the model. The texture-mapped 3-D head model is saved in VRML format The rotation angles $\alpha, \beta$ and $\gamma$ of the head model at the initial pose are set to zero degrees. We rotate the 


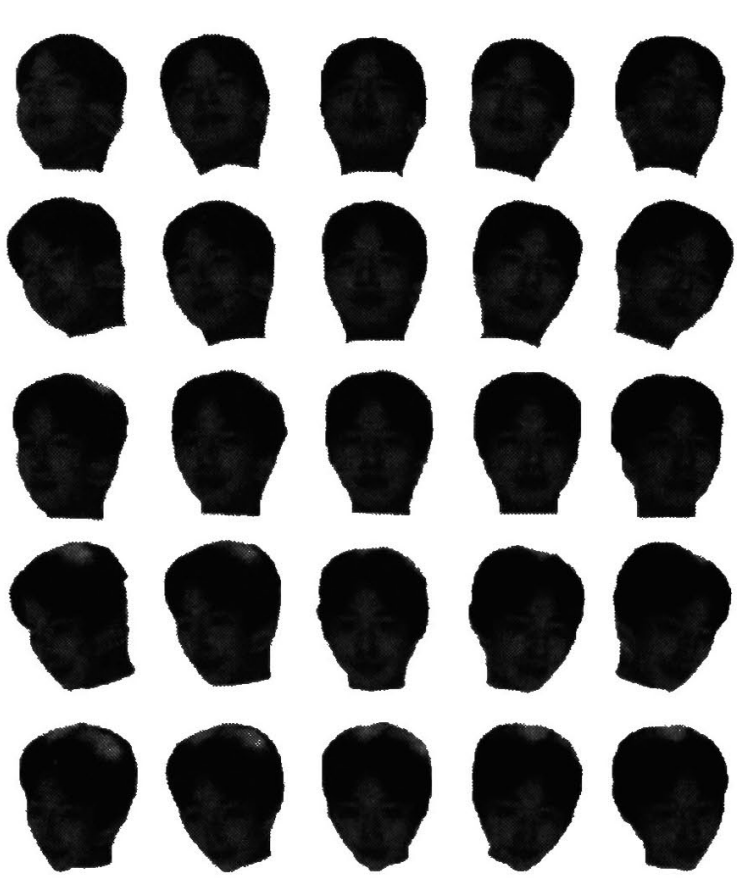

Fig. 7 Face with Different Orientation

head model by changing the value of $\alpha, \beta$ and $\gamma$ to produce CG heads with different poses, and then take stereo images at each pose using virtual stereo cameras. The function of virtual stereo cameras is realized by the VRML command. We set the virtual stereo cameras with same inner parameters and configuration as the real stereo camera we use. Sample heads are produced in the range where $\alpha$, $\beta$ and $\gamma$ increase from -30 degrees to +30 degrees by increments of 5 degrees. Fig. 7 shows part of the CG heads corresponding to 25 head poses, numbered as $1,2,3 \cdots, 25$ from the left top to the right down. The rotation angle of each pose is shown in Fig. 8 .

From the four extracted facial features, four feature triangles can be obtained. We observed that even when the head is in the same pose, detected pose value is different when using different triangles. One triangle may have a

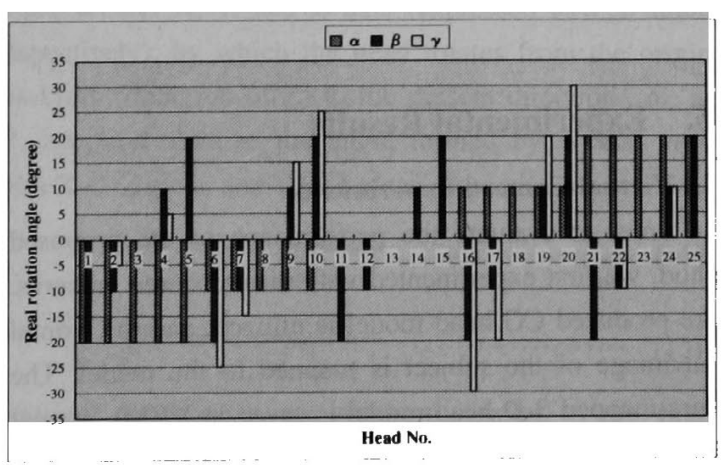

Fig. 8 Real Orientation of Face high accuracy at some poses, but will have a low accuracy at other poses. This can be explained by the analysis in section 4. Therefore, instead of using only one triangle, we calculate head pose from all of the four triangles, and use the average of the results from the four triangles as head pose output. Assuming that $\mathrm{V}_{1}, \mathrm{~V}_{2}, \mathrm{~V}_{3}, \mathrm{~V}_{4}$ are the results calculated from the triangles $P_{1}-P_{r}-M_{1}, P_{1}-P_{r}-M_{r}, P_{1}-M_{r}-M_{1}$ and $P_{r}-M_{r}-M_{1}$ respectively, then the result of the detected head pose $\mathrm{V}$ will be calculated as:

$$
V=\frac{1}{4} \sum_{i=1}^{4} V_{i}
$$

Fig.9 shows the detected pose of the sample heads, Fig. 10 shows the detection error at each pose. Fig. 11 gives a comparison between the errors of pose detection using each

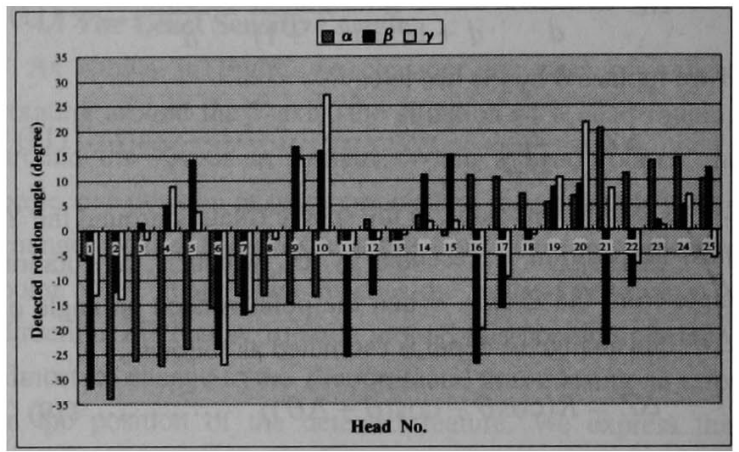

Fig. 9 Detected Orientation of Face

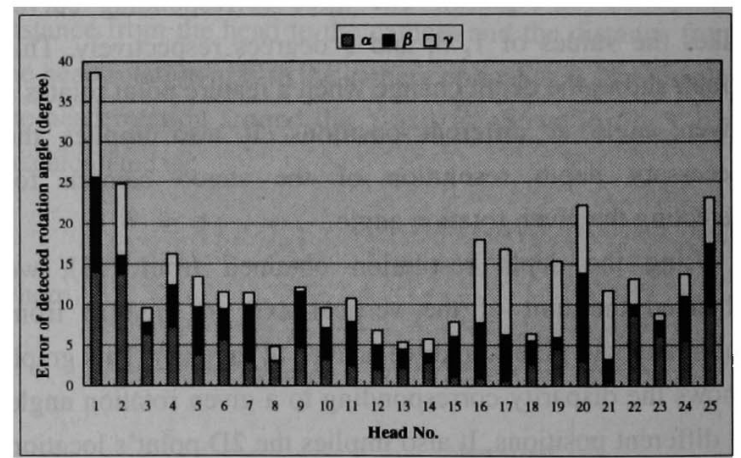

Fig. 10 Error of Estimated Orientation

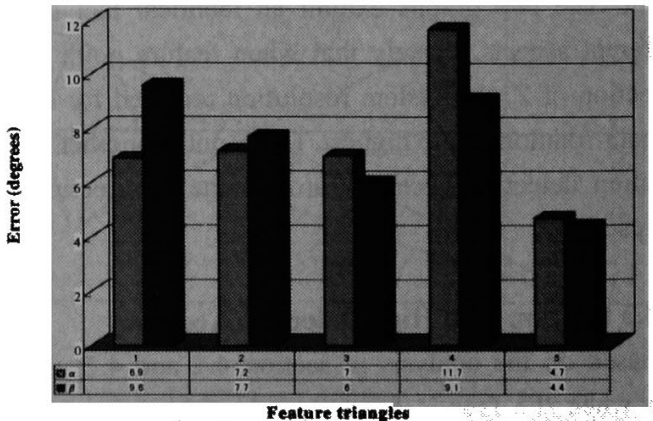

Fig. 11 Comparison of Detection Error

T. IEE Japan, Vol. 118-C, No. 10, '98 
triangle and using four triangles. Item 1,2,3 and 4 represent the detection error using triangle $P_{1}-P_{r}-M_{1}, P_{1}-P_{r}-M_{r}, P_{1}-M_{r}-M_{1}$ and $P_{r}-M_{r}-M_{1}$ respectively, and item5 represents the error of using the average of the four triangles. It is clear that the error arrived at in using four triangles is lower than that of using any single triangle.

When we consider the head in multiple poses, the average value of the pose detection error at each pose is defined as the average error. The average error can be a good description of the accuracy of a certain range. Table. 1 gives the average error distribution of detected rotation angles in four different ranges. It is clear that when head is not far away from the frontal direction, (i.e. rotation less than 20 degrees), the average detection error is low. This result is consistent with our analysis in section 4 .

Table 1. Average Error Distribution

\begin{tabular}{|c|c|c|c|}
\hline \multirow{2}{*}{$\begin{array}{l}\text { Absolute value of real angle } \\
\text { (degrees) }\end{array}$} & \multicolumn{3}{|c|}{ Average error } \\
\cline { 2 - 4 } & $\alpha$ & $\beta$ & $\gamma$ \\
\hline$[0,10]$ & 2.8 & 3.1 & 3.8 \\
\hline$[10,20]$ & 7.6 & 6.4 & 5.6 \\
\hline$[20,30]$ & 17.2 & 16.7 & 9.8 \\
\hline$[0,20]$ & 4.7 & 4.3 & 4.4 \\
\hline
\end{tabular}

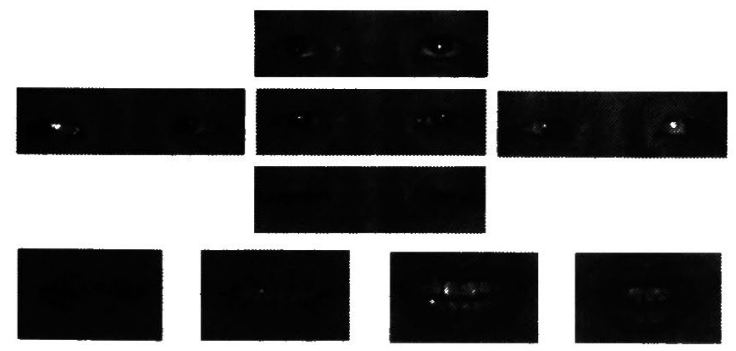

Fig. 12 Typical Facial Feature Movement

Even when the head pose remains unchanged, eyeball and mouth movement are possible, and thus make for errors in head pose detection. Fig. 12 shows some typical type of eyeball and mouth movement when the face is in a frontal orientation. Since the eyeballs always simutaneously move in parallel in a given direction, the disparity of eyeball variation is limited. Since the mouth moves when a person speaks, laughs, or has a change in expression, its movement is not easy to predict as that of the eyeball. Since we take the average of results from all triangles, the error caused by feature movement can be compensated to a limited degree. We performed an experiment that let eyeball or mouth move in the way as shown in Fig.12, but kept head pose unchanged ( $\alpha, \beta$ and $\gamma$ are zero). For comparison, the results with and without feature movement are both shown in table 2 .

Table 2. Error Caused by Feature Movement

\begin{tabular}{|l|c|c|}
\hline \multirow{2}{*}{} & \multicolumn{2}{|c|}{ error (degrees) } \\
\cline { 2 - 3 } & $\alpha$ & $\beta$ \\
\hline Eye movement & 4.7 & 4.6 \\
\hline Mouth movement & 9.5 & 4.2 \\
\hline No feature movement & 2.1 & 2.2 \\
\hline
\end{tabular}

\subsection{Experiments with Real Camera}

Our experimental system consisted of a Pentium $166 \mathrm{MHz}$ computer, two color video cameras (Sony XC-999 CCD), and two still image grabbers (Canopus V-port). Two cameras were spaced $200 \mathrm{~mm}$ in parallel as shown in Fig.4. The input image size was $640 \times 480$ pixels; the resolution of CCD sensor is 120 pixelmm. The subject was sitting about $130 \mathrm{~cm}$ away from the cameras, and the pictures were taken in front of a pre-arranged background. At present, pose detection is done off-line after capture of stereo images due to the properties of the image grabber that we use. We have tested 20 pairs of stereo images on the above computer, and the average processing time for each pair of stereo images has been $170 \mathrm{~ms}$, that is about $6 \mathrm{fps}$. It is obvious that when we adapt the image grabber for online processing and use computers with higher performance which are now readily available, the pose detection processing can be easily executed at a higher speed.

When using a real camera, errors of camera parameters, such as focal length, the lens distortion efficient, and distortion center, the relation between world coordinates and grabber frame coordinate and the like will directly influence the estimation result. In our experiment, camera calibration is performed using method[16].

Fig. 13 shows four frames of the facial image (the left one of the input stereo images) and the results of the detected head pose as represented by CG images. In our experiment, since we have no precision tools to measure the

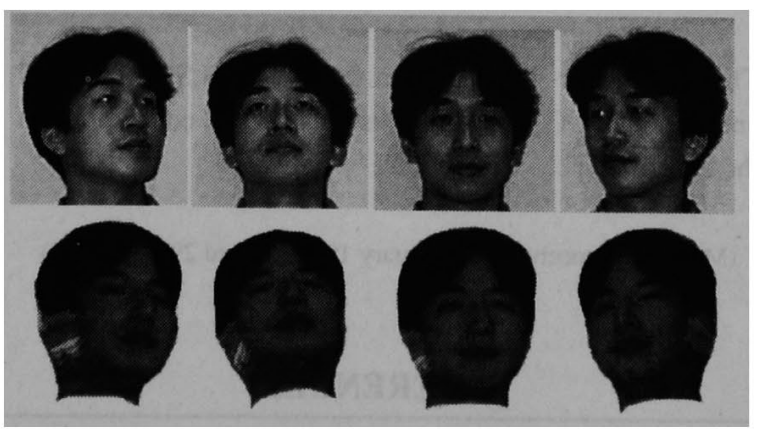

Fig. 13 Real Face (top row) and CG Face with the Estimated Orientation (down row) 
real head poses, only a coarse numerical analysis has been carried out. We let the subject take certain head pose, i.e. the value of $(\alpha, \beta, \gamma)$ became $(0,0,0),(0,-10,0),(0,-20,0)$, and the results are shown in Table 3 . It can be seen that the result is worse than that of using the CG image, and the reason may be found in errors of stereo camera calibration.

Table 3. A Coarse Numerical Analysis

\begin{tabular}{|c|c|c|c|}
\hline \multirow{2}{*}{$\begin{array}{l}\text { Practical pose } \\
\text { (degrees) }\end{array}$} & \multicolumn{3}{|c|}{ Detected pose (degrees) } \\
\cline { 2 - 4 } & $\alpha$ & $\beta$ & $\gamma$ \\
\hline$(0,0,0)$ & 4.5 & -5.3 & 2.1 \\
\hline$(0,-10,0)$ & 6.7 & -17.5 & 4.7 \\
\hline$(0,-20,0)$ & 10.2 & -33.7 & 9.6 \\
\hline
\end{tabular}

\section{Conclusion}

In this paper, we have proposed a method for estimating human facial orientation by the measurement of the 3-D position of facial features. Using a global-to-local feature detector and stereo vision, 3-D positions can be obtained accurately. Analytical and experimental results show that detection accuracy is high if the face does not rotate too far away from the frontal view. In our experiments using a virtual camera, when the head rotates less than twenty degree from the frontal view, average errors are less than five degrees. This is a good property for constructing a head pose based interaction system. However, when the face is obstructed by glasses or a beard, performance of the feature detector may degrade.

In practical situation, background changes may affect results, and our assumption in the paper may not necessarily be satisfied. As part of our continuing work in this area, we plan to develop a stronger and more effective facial detection method that can extract faces from complex backgrounds by employing multiple cues, such as face shape, skin color, and head motion.

\section{Acknowledgment}

The first author would like to thank Mr. M.SHIMANUKI and Mr. Yu GUO for helpful discussions and cooperation in the experiments.

(Manuscript received 25 February 1998; revised 29 June 1998)

\section{REFERENCES}

[1] T.E.Hutchinson, K.P.White et al. "Human-computer interaction using eye-gaze input", IEEE Transactions on
System, Man and Cybernetics, 19(6): pp.1527-1533, November/December 1989.

[2] A.Azarbayejani, A.Pentland et al. "Visually controlled graphics", IEEE Transactions on Pattern Analysis and Machine Intelligence, 15(6) pp.602-605 June 1993.

[3] R.Koch, "Dynamic 3-Dscene analysis through synthesis feedback control", IEEE Transactions on Pattern Analysis and Machine Intelligence, 15(6) pp.556-568 June 1993

[4] Ming Xu, T.Akatsuka, "Detecting head pose from stereo image sequence for active face recognition", Proceeding of Third IEEE international Conference on Automatic Face and Gesture Recognition, pp.82-87, 1998.

[5] A.Tukamoto, C.Lee, and S.Tsuji, "Detection and Tracking of Human Face with Synthesized Templates”, Proc. ACCV'93, pp.183-186, 1993.

[6] Kaori Suzuki et al. "Estimation of face orientation from shading images", Transaction of IEE Japan, Vol. 117-C, No.10 1997.

[7] Kenji Mase, Y.Watanabe, and Y.Suenaga, "Headreader: Realtime Motion Detection of Human Head from Image Sequence", IEICE Vol.J74-D-II pp.398-406 March 1991.

[8] K.Ohmura, A.Tomono, and Y.Kobayashi, "Method of Detecting Face Direction Using Image Processing for Human Face", Proceeding SPIE Visual Communication \& Image Processing '88 pp.625-632.

[9] Thanarat Horprasert, Yaser Yacoob, and Larry S.Davis, "Computing 3-D Head Orientation from a Monocular Image Sequence", Proceeding SPIE Vol. 2962, pp. 244-252, 25th AIPR Workshop: Emerging Applications of Computer Vision.

[10] Thomas Maurer, "Estimation of Face Position and Pose with Labeled Graphs", Proceeding of the BMVC 1996.

[11] Andrew Gee and Robert Cipolla, "Fast Visual Tracking By Temporal Consensus", Image and Vision Computing, 14(2), 105-114, 1996.

[12] Ryo Takamatsu, Yasuhiro Takada, and Makoto Sato, "Head posture estimation method for vision based human interface", Transaction of IEE Japan, Vol. 117-C, No. 101997.

[13] N.Otsu, "A threshold selection method from gray-level histograms", IEEE Trans. SMC-9 pp.62-66, 1979

[14] R.M.Haralick, S.R.Sternberg, and Xinhua Zhuang, "Image Analysis Using Mathematical Morphology", IEEE Transactions on Pattern Analysis and Machine Intelligence, Vol. PAMI-9, No.4, pp.532-550, 1987

[15] K. Deguti, "Images of 3D Space ---Mathematical geometry of Computer Vision”, SYOKODOU Press 1991.

[16] R.Y. Tsai, "A Versatile camera calibration technique for highaccuracy 3D machine vision metrology using off-the-shelf tv camera and lens", IEEE Journal of Robotics and Automation, 3(4): pp.323-344, Aug. 1987. 


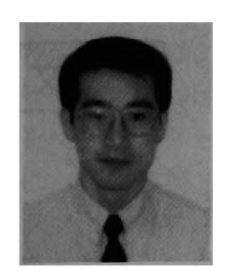

Ming XU (student member) received his B.E. degree from Tsinghua University, Beijing, China in 1989 and received his M.E. degree from Yamagata University, Yonezawa, Japan in 1992. He was an engineer in the Tsinghua Unisplendour Group, Beijing, China from 1992 to 1995 . He is now a Ph.D. candidate at the Engineering Faculty of Yamagata University.

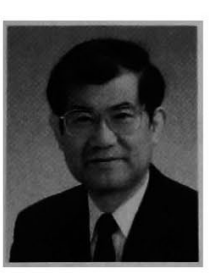

Takao AKATSUKA (nonmember) received his B.E., M.E., and Ph.D. from the University of Tokyo, in 1964, 1966 and 1974, respectively. From 1969 to 1974, he was an Instructor of the Department of Mathematical Engineering and Instrumentation Physics, University of Tokyo, and from 1974 to 1983, he was Assistant professor of Medical Engineering at the Univ. of Tsukuba. $\mathrm{He}$ is currently Professor of Electrical and Information Engineering Department of Engineering Faculty, Yamagata University, Yonezawa. His interests are imaging and image understanding technology. 